\title{
MOMENTS OF THE LENGTH OF LINE SEGMENTS IN HOMOGENEOUS PLANAR STIT TESSELLATIONS
}

\author{
CHRISTOPH THÄLE \\ Department of Mathematics, University of Fribourg, CH-1700 Fribourg, Switzerland \\ e-mail: christoph.thaele@unifr.ch \\ (Accepted February 20, 2009)
}

\begin{abstract}
Homogeneous planar tessellations stable under iteration (STIT tessellations) are considered. Using recent results about the joint distribution of direction and length of the typical $I-, K$ - and $J$-segment we prove closed formulas for the first, second and higher moments of the length of these segments given their direction. This especially leads to the mean values and variances of these quantities and mean value relations as well as general moment relationships. Moreover, the relation between these mean values and certain conditional mean values (and also higher moments) is discussed. The results are also illustrated for several examples.
\end{abstract}

Keywords: conditional distribution, iteration (nesting), linear segments, mean value relation, moments, random tessellation, stability, stochastic geometry.

\section{INTRODUCTION}

Random tessellations are nowadays used for modeling several structures which arise for example in material sciences, biology and medical sciences. The most popular models are Poisson line or plane tessellations or Poisson-Voronoi tessellations (see Stoyan et al., 1995). These models are on the one hand side mathematically feasible and useful in practice on the other. More complex models can be obtained by applying certain operations on given tessellations. These are for example superposition or iteration. The latter operation leads to a relatively new model for random tessellations, the so-called STIT model. The name STIT refers to their characteristic property: they are stable under iteration. This property will be explained in some detail in the next section.

Several geometric quantities were calculated for this model by now. This includes mean value formulas in $2 \mathrm{D}$ and $3 \mathrm{D}$ and length distributions of several linear segments. For the latter Mecke et al. (2007) calculated also first and second moments, but only for the isotropic case. They especially observed that the variance of the length of the so-called typical $I$ segment is infinite. The same observation was made in Thäle (2008) also for two anisotropic examples. It is one aim of this short paper to undertake a deeper study of this phenomenon. We will show that the variance (and also other higher moments) of the length of the typical $I$-segment does not exist for any homogeneous planar STIT tessellation, which shows, that these linear segments are in some sense very long. In contrast to this result we will be able to show that for the length of the typical $K$ - and $J$-segment all moments exist. Moreover, we will derive explicit formulas for all moments of the quantities in question for arbitrary homogeneous planar STIT tessellations.

The other aim of the paper is the study of mean value relations. In particular we are interested in mean values for the lengths of the typical $I-, K$ - and $J$ segment having a fixed direction and their relation to the mean values without the directional conditioning (this could be of some interest for stereological questions). At this point the directional distribution of the tessellation plays an important role and we will be able to extend the mean value formulas to the case, where the typical $I-, K$ - or $J$-segment is replaced by the typical $I-, K$ - or $J$-segment with a fixed given direction. Moreover, the known formulas can be recovered by averaging over all possible directions. This observation is a new feature of STIT tessellations, which can only be observed, when the anisotropic case is studied and this was not investigated until the recent works Mecke (2008) and Thäle (2008). We will furthermore obtain a general moment relationship for the conditional and unconditional length distribution of the segments. Our results are demonstrated on several concrete examples, in particular we consider the isotropic case and confirm again the results of Mecke et al. (2007). Also the new rectangular case and a case with unequal weights is discussed.

\section{PLANAR STIT TESSELLATIONS}

By a planar tessellation we mean a subdivision of the plane into a locally finite union of convex polygons, which intersect only in their boundaries. 
The family of such tessellations will be denoted by $\mathscr{T}$. Such tessellations can also be described as the union of their cell boundaries. This allows us to consider a tessellation as a closed subset of $\mathbb{R}^{2}$. We will follow this path here. Denoting by $\mathfrak{T}$ the restriction of Matheron's $\sigma$-algebra ( $c f$. Stoyan et al., 1995) to $\mathscr{T}$, we call a random variable $\Phi$ with values in the measurable space $[\mathscr{T}, \mathfrak{T}]$ a random planar tessellation. For $x \in \mathbb{R}^{2}$ we denote by $T_{x}$ the translation of $\mathbb{R}^{2}$ by the vector $-x$. $T_{x}$ induces also an operation on the space of tessellations, also denoted by $T_{x}$, by $T_{x} \Phi=\Phi-x$. We say that a random tessellation $\Phi$ is homogeneous (or stationary), if $T_{x} \Phi$ has the same distribution as $\Phi$ for all $x \in \mathbb{R}^{2}$. The law of a homogeneous tessellation is also called homogeneous.

We fix now two random planar tessellations $\Phi$ and $\Psi$ with law $P$ and $Q$, respectively. The cells of $\Phi$ are denoted by $C(\Phi)$. We associate now to each cell $p \in C(\Phi)$ independently a random tessellation $\Psi_{p}$ with law $Q$. Now define a random tessellation $\Phi \odot \Psi$ by

$$
\Phi \odot \Psi:=\Phi \cup \bigcup_{p \in C(\Phi)}\left(p \cap \Psi_{p}\right),
$$

the law of which is denoted by $P \boxplus Q$. It was shown in Mecke et al. (2008b) that if $\Phi$ is a homogeneous tessellation and $Q$ is also homogeneous then $\Phi \odot \Psi$ is a homogeneous random tessellation, too. We call a homogeneous random tessellation $\Phi$ stable with respect to iteration (STIT for short), if $2(\Phi \odot \Phi)$ has the same law as $\Phi$ itself (this is equivalent to the definition used for example in Nagel and Weiss (2005), see Mecke, 2008).

The existence of such tessellations was shown in Nagel and Weiss (2005) together with a construction in a bounded window for arbitrary dimensions. A global construction of planar STIT tessellations was recently presented in Mecke et al. (2008a).

Denote by $[\mathscr{H}, \mathfrak{H}]$ the measurable space of lines through the origin. By the direction of an arbitrary line $g$ we mean the unique parallel line $r(g) \in \mathscr{H}$. For a line segment $s$ denote the line $g$ containing $s$ by $g(s)$. Then the direction $r(s)$ of $s$ can be defined as $r(s):=r(g(s)) \in \mathscr{H}$. For a planar tessellation $\Phi$ we introduce now a directional measure $\kappa$ as follows: For $B \in \mathfrak{H}$ we consider the family $\Phi_{B}$ of edges with direction in $B$. The mean length of these edges per unit area (note that this is a well defined quantity, since $\Phi$ is homogeneous) will be denoted by $L_{A}(B)$. By the relation $\kappa: \mathfrak{H} \rightarrow[0, \infty): B \mapsto L_{A}(B)$ we get a measure $\kappa$ on $[\mathscr{H}, \mathfrak{H}]$, the directional measure of $\Phi$. If we denote by $L_{A}$ the edge length intensity, i.e., the mean total edge length per unit area, of $\Phi$, we can write $\kappa$ also as $\kappa=L_{A} \vartheta$ for some probability measure $\vartheta$ on $[\mathscr{H}, \mathfrak{H}]$, since $\kappa(\mathscr{H})=L_{A}$. The latter is called directional distribution of $\Phi$. We assume in this paper, that $\kappa$ (or equivalently $\vartheta$ ) is concentrated on more than a single direction. This ensures the existence of of a STIT tessellation with this measure as its directional measure or distribution ( $c f$. Nagel and Weiss, 2005). Having in mind these notions and notations we can define the rose of intersections of $\kappa$ as

$$
s_{\kappa}: \mathscr{H} \rightarrow(0, \infty): h \mapsto \int_{\mathscr{H}}|\sin \angle(h, \tilde{h})| \mathrm{d} \kappa(\tilde{h}) .
$$

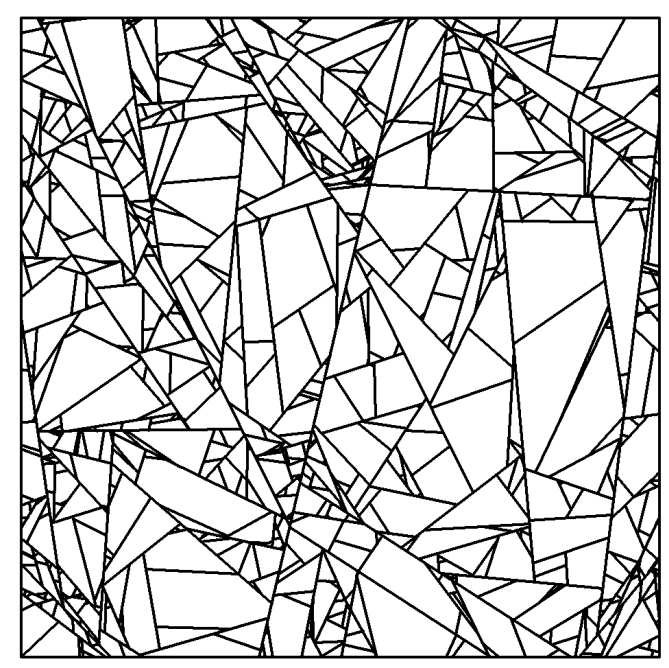

Fig. 1. Realization of an isotropic STIT tessellation.

We summarize now the most important features of planar STIT tessellations $\Phi$ with directional measure $\kappa$ :

1. The intersection of $\Phi$ with an arbitrary line $g$ (not necessarily through the origin) is a homogeneous Poisson point process on $g$. It has intensity $s_{\kappa}(r(g))(c f$. Nagel and Weiss, 2005).

2. The interior of the typical cell (this is a cell with Palm shape distribution, see Stoyan et al., 1995) has the same distribution as the interior of the typical cell of a Poisson line tessellation with the same directional measure (cf. Nagel and Weiss, 2003).

3. The nodes (vertices) have $T$-shape, i.e., from each node we have three emanating edges and two of them are collinear ( $c f$. Nagel and Weiss, 2003).

4. The cells of $\Phi$ are not in a face-to-face position, see Fig. 1 ( $c f$. Nagel and Weiss, 2005).

More results about STIT tessellations can be found in Nagel and Weiss (2003), Nagel and Weiss (2005), Nagel and Weiss (2006),Mecke et al. (2007), Mecke et al. (2008a), Mecke et al. (2008b), Thäle (2008), Thäle (2009). 


\section{RECENT RESULTS FOR $I-, K-$ AND J-SEGMENTS}

Mackisack and Miles (1996) introduced the notion of $I$-, $K$ - and $J$-segments and showed that their analysis can be fruitful, especially in the case of tessellations which are not face-to-face. A $K$-segment they called every line segment of the tessellation without any vertex in its relative interior. A $J$-Segment is a face of a cell and an $I$-segment is the union of connected and collinear $K$-segments, which cannot be enlarged by another $K$-segment.

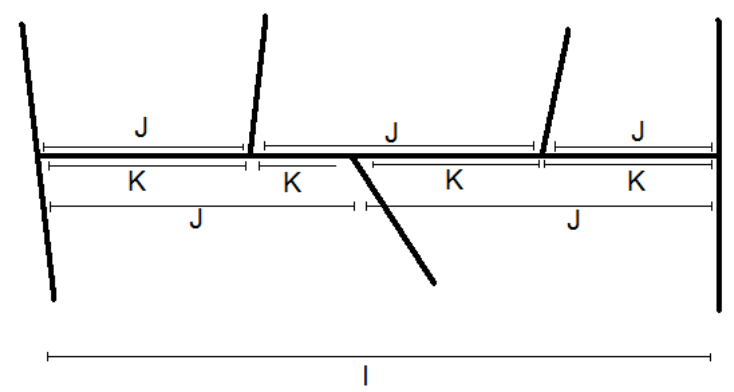

Fig. 2. Different types of linear segments in a planar tessellation.

The investigation of the length distribution of these segments for planar STIT tessellations starts in Mecke et al. (2007) for the isotropic case. The authors of this paper were able to calculate explicitly the densities of the length distribution of the segments and by integration their mean values and second moments (compare with the isotropic example below).

In his paper, Mecke (2008) starts the analysis of the anisotropic case. Here, in contrast to the isotropic case, one has to take also into account the direction of the segments. Thus, he considered the joint distribution of direction and length for the case of $I$-segments. His approach was completed by the consideration of the typical $K$ - and $J$-segment in Thäle (2008) (here typical should be understood in the Palm sense, where the Palm distribution with respect to the segment midpoints is considered).

We like to summarize the results now. Let therefore $\Phi$ be a homogeneous planar STIT tessellation with directional measure $\kappa$. The rose of intersections of $\kappa$ is again given by Eq. 1. The directional distribution of $\Phi$ will be denoted by $\vartheta$. We further define the constant

$$
\zeta_{\kappa}:=\int_{\mathscr{H}} \int_{\mathscr{H}}|\sin \angle(h, \tilde{h})| \mathrm{d} \kappa(h) \mathrm{d} \kappa(\tilde{h}) .
$$

The joint distribution of direction and length of the typical $X$-segment, $X \in\{I, J, K\}$, is a probability measure on $\mathscr{H} \times(0, \infty)$ having density $d_{X}$ with respect to the product measure $\kappa \times \mathscr{L}_{+}$, where $\mathscr{L}_{+}$is the Lebesgue measure restricted to $(0, \infty)$. Then we have (Theorem 12 in Mecke, 2008, Corollary 3.4 and Corollary 3.6 in Thäle, 2008):

$$
\begin{aligned}
& d_{I}:(h, x) \mapsto \frac{2}{\zeta_{\mathcal{K}} s_{\mathcal{K}}(h)} \int_{0}^{s_{\mathcal{K}}(h)} t^{2} \mathrm{e}^{-t x} \mathrm{~d} t, \\
& d_{K}:(h, x) \mapsto \frac{2}{3 \zeta_{\kappa}} s_{\mathcal{K}}^{2}(h) \int_{1}^{2} t^{2} \mathrm{e}^{-s_{K}(h) t x} \mathrm{~d} t, \\
& d_{J}:(h, x) \mapsto \frac{s_{\mathcal{K}}(h)}{\zeta_{\kappa}} \int_{x}^{\infty} \mathrm{e}^{-s_{\mathcal{K}}(h) t} \mathrm{~d} t .
\end{aligned}
$$

We consider now the marked point process $\alpha_{X}=$ $\left\{y_{k}, h_{k}\right\}_{k \in \mathbb{N}}, X \in\{I, J, K\}$, of $X$-segment midpoints $y_{k}$, where the marks $h_{k} \in \mathscr{H}$ are given by the direction of the $X$-segment through $y_{k}$. The typical $X$-segment in direction $h \in \mathscr{H}$ of the tessellation $\Phi$ can now be defined as the line segment containing the origin, where $\Phi$ is considered under the Palm distribution $P^{0, h}$. (For the general theory of Palm distributions for marked point processes see for example Stoyan et al., 1995).

From the formulas above we can conclude the following integral representations for the conditional densities $d_{I \mid h}, d_{J \mid h}$ and $d_{K \mid h}$ of the length distributions of the typical $I$-, $K$ - and $J$-segment in direction $h \in \mathscr{H}$, since the density of the direction with respect to the directional measure $\kappa$ of the typical $I-, K$ - and $J$ segment is given by $s_{K}(\cdot) / \zeta_{K}$ in each case ( $c f$. Mecke, 2008; Thäle, 2008):

$$
\begin{aligned}
d_{I \mid h}(x) & =\frac{2}{s_{K}^{2}(h)} \int_{0}^{s_{K}(h)} t^{2} \mathrm{e}^{-t x} \mathrm{~d} t \\
d_{K \mid h}(x) & =\frac{2}{3} s_{K}(h) \int_{1}^{2} t^{2} \mathrm{e}^{-s_{K}(h) t x} \mathrm{~d} t \\
d_{J \mid h}(x) & =\int_{x}^{\infty} \mathrm{e}^{-s_{K}(h) t} \mathrm{~d} t
\end{aligned}
$$

The densities written in this form (and not explicitly without an integral) will turn out to be very powerful and lead to short proof in the next section.

\section{MAIN RESULTS}

We consider a homogeneous planar STIT tessellation $\Phi$ with directional measure $\kappa$. Denote the rose of intersections of $\kappa$ again by $s_{\kappa}$ and fix some direction $h \in \mathscr{H}$. The typical $I-, K$ - and $J$-segment with direction $h$ will be denoted by $I^{0, h}, K^{0, h}$ and $J^{0, h}$, respectively. The (euclidean) length of $I^{0, h}, K^{0, h}$ and $J^{0, h}$ is denoted by $L\left(I^{0, h}\right), L\left(K^{0, h}\right)$ and $L\left(J^{0, h}\right)$, 
respectively. For $k=1,2,3, \ldots$ we define now the $k$ moments

$$
\begin{aligned}
I_{k}(h) & :=\mathbb{E}\left|L\left(I^{0, h}\right)\right|^{k}, \\
K_{k}(h) & :=\mathbb{E}\left|L\left(K^{0, h}\right)\right|^{k}, \\
J_{k}(h) & :=\mathbb{E}\left|L\left(J^{0, h}\right)\right|^{k} .
\end{aligned}
$$

\section{TYPICAL I-SEGMENTS}

We start our analysis with the typical $I$-segment $I^{0, h}$ in direction $h \in \mathscr{H}$. First observe that integration by parts yields for $t>0$

$$
\int_{0}^{\infty} x^{k} \mathrm{e}^{-t x} d x=t^{-k-1} \Gamma(k+1)
$$

where $\Gamma(\cdot)$ is Euler's Gamma-function. We can now use Fubini's theorem and formula Eq. 2 to obtain

$$
\begin{aligned}
& I_{k}(h)=\int_{0}^{\infty} x^{k} \mathrm{~d}_{I \mid h}(x) \mathrm{d} x \\
& =\int_{0}^{\infty} x^{k}\left[\frac{2}{s_{\mathcal{K}}(h)^{2}} \int_{0}^{s_{K}(h)} t^{2} \mathrm{e}^{-t x} \mathrm{~d} t\right] \mathrm{d} x \\
& =\frac{2}{s_{K}(h)^{2}} \int_{0}^{s_{K}(h)} t^{2}\left[\int_{0}^{\infty} x^{k} \mathrm{e}^{-t x} \mathrm{~d} x\right] \mathrm{d} t \\
& =\frac{2}{s_{K}(h)^{2}} \Gamma(k+1) \int_{0}^{s_{K}(h)} t^{2} t^{-k-1} \mathrm{~d} t \\
& =\frac{2}{s_{\kappa}(h)^{2}} \Gamma(k+1) \int_{0}^{s_{K}(h)} t^{-k+1} \mathrm{~d} t \\
& =\left\{\begin{array}{lll}
\frac{2}{s_{K}(h)} & : & k=1, \\
+\infty & : & k \geq 2 .
\end{array}\right.
\end{aligned}
$$

This means for the length of the typical $I$-segment in any direction $h$, that only the first moment exists. All higher moments are infinite.

\section{TYPICAL K-SEGMENTS}

We continue with the typical $K$-segment $K^{0, h}$ with direction $h \in \mathscr{H}$. First observe that

$$
\int_{0}^{\infty} x^{k} \mathrm{e}^{-s t x} \mathrm{~d} x=(s t)^{-k-1} \Gamma(k+1)
$$

for all $s, t>0$. We use now formula Eq. 3 and again Fubini's theorem to obtain

$$
\begin{aligned}
K_{k}(h) & =\int_{0}^{\infty} x^{k} d_{K \mid h}(x) \mathrm{d} x \\
& =\int_{0}^{\infty} x^{k}\left[\frac{2}{3} s_{K}(h) \int_{1}^{2} t^{2} \mathrm{e}^{-s_{K}(h) t x} \mathrm{~d} t\right] \mathrm{d} x \\
& =\frac{2}{3} s_{\mathcal{K}}(h) \int_{1}^{2} t^{2}\left[\int_{0}^{\infty} x^{k} \mathrm{e}^{-s_{K}(h) x} \mathrm{~d} x\right] \mathrm{d} t \\
& =\frac{2}{3} s_{\mathcal{K}}(h)^{-k} \Gamma(k+1) \int_{1}^{2} t^{2} t^{-k-1} \mathrm{~d} t \\
& =\frac{2}{3 s_{K}(h)^{k}} \Gamma(k+1) \int_{1}^{2} t^{-k+1} \mathrm{~d} t \\
& = \begin{cases}\frac{2}{3 s_{K}(h)^{k}} \Gamma(k+1) \frac{2^{k}-4}{2^{k}(k-2)}: & : \quad k=2 . \\
\frac{4}{3 s_{K}(h)^{2}} \ln 2 & \end{cases}
\end{aligned}
$$

This shows, that in contrast to the previous case all moments of the length of the typical $K$-segment with direction $h$ exist. We also obtained a closed formula for them.

\section{TYPICAL J-SEGMENTS}

This case is much easier, since $L\left(J^{0, h}\right)$ is exponentially distributed with parameter $s_{K}(h)$, because of the Poisson typical cells of STIT tessellations (see the key properties in the section on planar STIT tessellations). Thus, we obtain

$$
J_{k}(h)=\mathbb{E}\left|L\left(J^{0, h}\right)\right|^{k}=\frac{\Gamma(k+1)}{s_{\kappa}(h)^{k}} .
$$

We see that also in the case of the typical $J$ segment in direction $h$ all moments exist. We like to remark that the result can also be obtained by a direct calculation using formula Eq. 4.

Note that in the above formulas for $I_{k}(h), K_{k}(h)$ and $J_{k}(h), \Gamma(k+1)$ could be replaced by $k$ !.

\section{MEAN VALUES AND VARIANCES}

We can especially apply our results to compute the mean values and the variances of $L\left(I^{0, h}\right), L\left(K^{0, h}\right)$ and $L\left(J^{0, h}\right)$. We consider therefore a homogeneous planar STIT tessellation with directional measure $\kappa$ and fix a direction $h \in \mathscr{H}$. Then we obtain 


$$
\begin{aligned}
\mathbb{E} L\left(I^{0, h}\right) & =\frac{2}{s_{\mathcal{K}}(h)}, \\
\mathbb{V} L\left(I^{0, h}\right) & =+\infty, \\
\mathbb{E} L\left(K^{0, h}\right) & =\frac{2}{3 s_{\mathcal{K}}(h)}, \\
\mathbb{V} L\left(K^{0, h}\right) & =\frac{2}{3 s_{\mathcal{K}}(h)^{2}}\left(2 \ln 2-\frac{1}{3}\right), \\
\mathbb{E} L\left(J^{0, h}\right) & =\frac{1}{s_{\mathcal{K}}(h)}, \\
\mathbb{V} L\left(J^{0, h}\right) & =\frac{1}{s_{\mathcal{K}}(h)^{2}},
\end{aligned}
$$

by using the formula $\mathbb{V} X=\mathbb{E} X^{2}-(\mathbb{E} X)^{2}$ for real valued random variables $X$.

\section{MOMENT RELATIONSHIPS}

Recall that $s_{\kappa}(\cdot) / \zeta_{\kappa}$ is the density of the direction of the typical $I-, J$ - and $K$-segment segment $I^{0}, K^{0}, J^{0}$ wrt. $\kappa$ (cf. Mecke, 2008; Thäle, 2008) and the relation $\kappa(\mathscr{H})=L_{A}$. We obtain the following mean values for the length of the typical $I-, J$ - and $K$-segment:

$$
\begin{aligned}
& \mathbb{E} L\left(I^{0}\right)=\mathbb{E}_{h}\left(\mathbb{E} L\left(I^{0, h}\right)\right)=\frac{2 L_{A}}{\zeta_{\kappa}}, \\
& \mathbb{E} L\left(K^{0}\right)=\mathbb{E}_{h}\left(\mathbb{E} L\left(K^{0, h}\right)\right)=\frac{2 L_{A}}{3 \zeta_{\kappa}}, \\
& \mathbb{E} L\left(J^{0}\right)=\mathbb{E}_{h}\left(\mathbb{E} L\left(J^{0, h}\right)\right)=\frac{L_{A}}{\zeta_{\kappa}},
\end{aligned}
$$

where $\mathbb{E}_{h}$ denotes the average over all directions $h$. For example

$$
\mathbb{E} L\left(I^{0}\right)=\int_{\mathscr{H}} \frac{2}{s_{\kappa}(h)} \cdot \frac{s_{\kappa}(h)}{\zeta_{\kappa}} d \kappa(h)=\frac{2}{\zeta_{\kappa}} \kappa(\mathscr{H})=\frac{2 L_{A}}{\zeta_{\kappa}} .
$$

Note that these mean values are the same as the one computed in Nagel and Weiss (2006), but there the constant $\zeta=\zeta_{\kappa} / L_{A}^{2}$ was used instead of $\zeta_{\kappa}$.

These formulas imply the following mean value relations:

$$
\mathbb{E} L\left(I^{0, h}\right)=3 \mathbb{E} L\left(K^{0, h}\right)=2 \mathbb{E} L\left(J^{0, h}\right)
$$

and

$$
\mathbb{E} L\left(I^{0}\right)=3 \mathbb{E} L\left(K^{0}\right)=2 \mathbb{E} L\left(J^{0}\right) .
$$

The relations Eq. 8 confirm earlier results from the paper Nagel and Weiss (2006), which were obtained by quite different methods. Beside these mean value relations we obtain the following relationship of the variances of the typical $K$ - and $J$-segment with direction $h \in \mathscr{H}$ :

$$
\begin{aligned}
\mathbb{V} L\left(K^{0, h}\right) & =\frac{2}{3}\left(2 \ln 2-\frac{1}{3}\right) \mathbb{V} L\left(J^{0, h}\right) \\
& \approx 0.70196 \cdot \mathbb{V} L\left(J^{0, h}\right) .
\end{aligned}
$$

For the higher moments $K_{k}(h)$ and $J_{k}(h), k \geq 3$, we get the following general relation for the conditional length distributions:

$$
K_{k}(h)=\frac{2}{3} \cdot \frac{2^{k}-4}{2^{k}(k-2)} J_{k}(h)
$$

for any $h \in \mathscr{H}$. An analogous formula can also be obtained for the unconditioned moments again simply by integration:

$$
\mathbb{E}\left|L\left(K^{0}\right)\right|^{k}=\frac{2}{3} \cdot \frac{2^{k}-4}{2^{k}(k-2)} \mathbb{E}\left|L\left(J^{0}\right)\right|^{k} .
$$

Note that

$$
\lim _{k \rightarrow \infty} \frac{2}{3} \cdot \frac{2^{k}-4}{2^{k}(k-2)}=0 .
$$

Comment: One can conclude from Eq. 8 the fact, that the mean number of nodes in the relative interior of the typical $I$-segment equals 2 and $1 / 2$ for the typical $J$-segment. It will be shown in the forthcoming paper (Thäle, 2009), that for STIT tessellations in $\mathbb{R}^{d}, d \geq 2$, the mean number of nodes in the relative interior of the typical $I$-segment equals $d$ and $(d-1) / 2$ for the typical $J$-segment $(I-, J$ - and $K$-segments of higher dimensional STIT tessellations are now considered for the 1-skeleton; the definition remains the same).

\section{EXAMPLES}

\section{THE ISOTROPIC CASE}

We consider in this section homogeneous and isotropic planar STIT tessellations with edge length intensity $\pi / 2$. In this case we can calculate the distribution functions $F_{I}, F_{K}$ and $F_{J}$ of the length of the typical $I$-, $K$ - and $J$-segment, respectively, using formulas Eqs. 2-4. We obtain

$$
\begin{aligned}
& F_{I}(x)=1-\frac{2}{x^{2}}\left(1-(1+x) \mathrm{e}^{-x}\right), \\
& F_{K}(x)=1-\frac{2}{3 x^{2}}\left(1+x-(1+2 x) \mathrm{e}^{-x}\right) \mathrm{e}^{-x}, \\
& F_{J}(x)=1-\mathrm{e}^{-x}
\end{aligned}
$$

which confirms the results of Mecke et al. (2007), especially Theorem 2 and 4 therein. For the densities $p_{I}(x), p_{K}(x)$ and $p_{J}(x)$ we get

$$
\begin{aligned}
& p_{I}(x)=\frac{4}{x^{3}}\left(1-\left(1+x+\frac{x^{2}}{2}\right) \mathrm{e}^{-x}\right), \\
& p_{K}(x)=\frac{4}{3 x^{3}}\left(\left(1+x+\frac{x^{2}}{2}\right) \mathrm{e}^{-x}\right. \\
& \left.-\left(1+2 x+2 x^{2}\right) \mathrm{e}^{-2 x}\right), \\
& p_{J}(x)=\mathrm{e}^{-x} .
\end{aligned}
$$




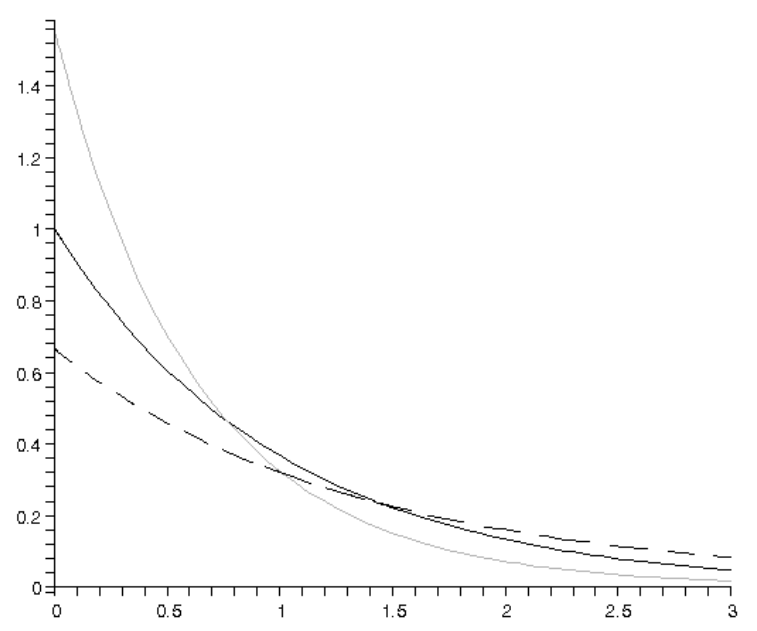

Fig. 3. Densities $p_{I}$ (black), $p_{K}$ (grey) and $p_{J}$ (dashed) in the isotropic case.

For the mean values and variances we have in this case

$$
\mathbb{E} L\left(I^{0}\right)=2, \quad \mathbb{E} L\left(K^{0}\right)=\frac{2}{3}, \quad \mathbb{E} L\left(J^{0}\right)=1
$$

and

$$
\begin{aligned}
\mathbb{V} L\left(I^{0}\right) & =+\infty, \\
\mathbb{V} L\left(K^{0}\right) & =\frac{4}{3}\left(2 \ln 2-\frac{1}{3}\right), \\
\mathbb{V} L\left(J^{0}\right) & =1
\end{aligned}
$$

from Eqs. 5-7, which confirms Eq. 8 and the earlier results from Nagel and Weiss (2006).

Note that in the isotropic case the length and the direction of the typical $I$-, $K$ - and $J$-segment are independent.

\section{THE RECTANGULAR CASE}

We consider a homogeneous planar STIT tessellation $\Phi$ with directional distribution

$$
\vartheta(h)=\left\{\begin{array}{lll}
\frac{1}{2} & : & h \text { is the } x \text { - or } y \text {-axis } \\
0 & : & \text { else }
\end{array}\right.
$$

and edge length intensity 1 . The cells of such a tessellation $\Phi$ are rectangles with probability one (a similar case was investigated in Mackisack and Miles (1996) but not for STIT tessellations of course).

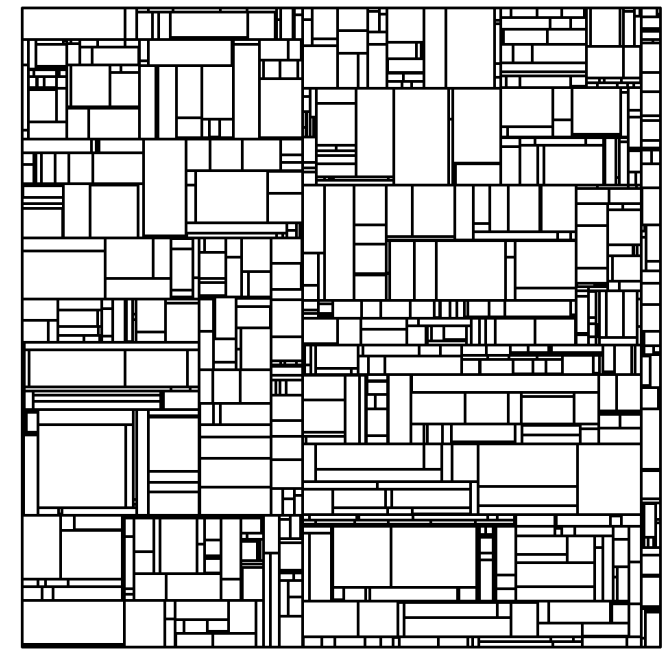

Fig. 4. Realization of a rectangular STIT tessellation.

From Eqs. 2-4 we calculate again the densities $p_{I}(x), p_{K}(x), p_{J}(x)$ of the length distribution of the typical $I-, K$ - and $J$-segment with direction in $x$ - or $y$ axis, respectively:

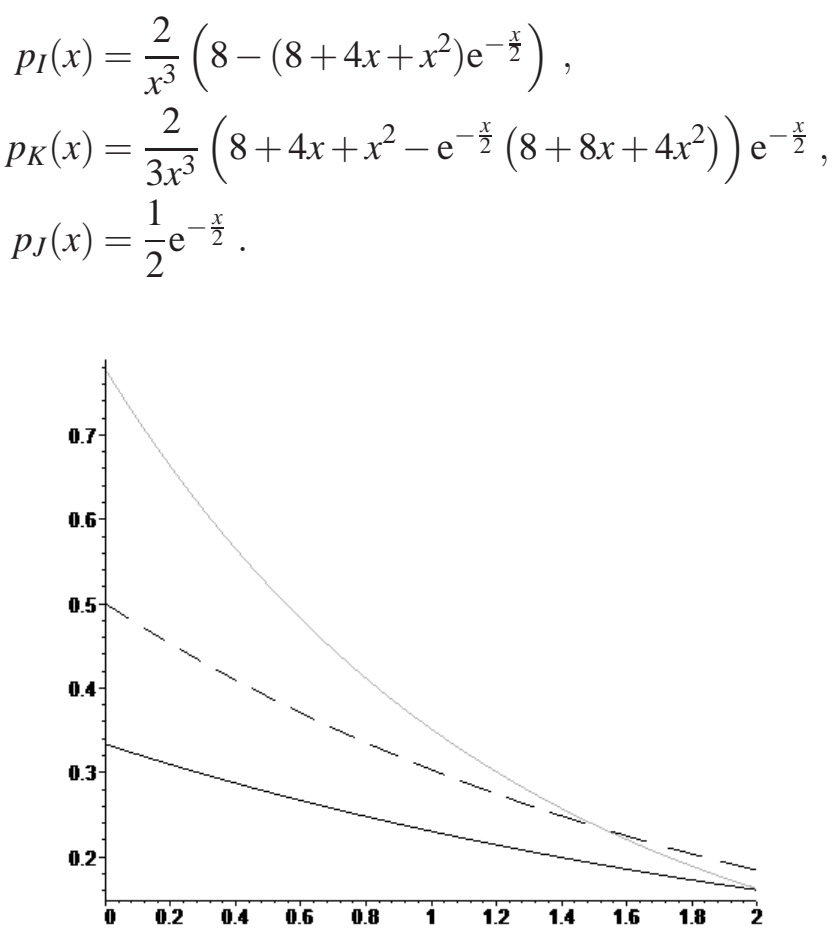

Fig. 5. Densities $p_{I}$ (black), $p_{K}$ (grey) and $p_{J}$ (dashed) in the rectangular case.

For the mean values and variances we obtain

$$
\mathbb{E} L\left(I^{0, h}\right)=4, \quad \mathbb{E} L\left(K^{0, h}\right)=\frac{4}{3}, \quad \mathbb{E} L\left(J^{0, h}\right)=2
$$

and 


$$
\begin{aligned}
\mathbb{V} L\left(I^{0, h}\right) & =+\infty \\
\mathbb{V} L\left(K^{0, h}\right) & =\frac{16}{3}\left(2 \ln 2-\frac{1}{3}\right) \\
\mathbb{V} L\left(J^{0, h}\right) & =4
\end{aligned}
$$

for both directions $h(x$ and $y)$. Furthermore we have

$$
\begin{aligned}
\mathbb{E} L\left(I^{0}\right) & =\frac{1}{2}\left(\mathbb{E} L\left(I^{0, x}\right)+\mathbb{E} L\left(I^{0, y}\right)\right)=4, \\
\mathbb{E} L\left(K^{0}\right) & =\frac{1}{2}\left(\mathbb{E} L\left(K^{0, x}\right)+\mathbb{E} L\left(K^{0, y}\right)\right)=\frac{4}{3}, \\
\mathbb{E} L\left(J^{0}\right) & =\frac{1}{2}\left(\mathbb{E} L\left(J^{0, x}\right)+\mathbb{E} L\left(J^{0, y}\right)\right)=2 .
\end{aligned}
$$

\section{A CASE WITH UNEQUAL WEIGHTS}

At the end we like to discuss an example with unequal weights. Consider therefore a directional distribution $\vartheta$, which has the following weights on three directions

$$
\vartheta(h)=\left\{\begin{array}{lll}
\frac{1}{2}: & h=x \text {-axis } \\
\frac{1}{3}: & h=\{x=y\}, \text { the diagonal } \\
\frac{1}{6}: & h=y \text {-axis }
\end{array}\right.
$$

The first direction is called $D_{1}$, the second $D_{2}$ and the third $D_{3}$ for abbreviation. We further assume that the edge length intensity $L_{A}$ is 1 . On can now calculate the 9 different densities $p_{X, D_{i}}(\cdot)$ for $X \in$ $\{I, J, K\}$ and $i=1,2,3$, but we do not give the explicit formulas here. Instead we concentrate on several mean value relations: For the mean values and variances in direction $D_{1}$ we have

$$
\mathbb{E} L\left(I^{0, D_{1}}\right)=4, \quad \mathbb{E} L\left(K^{0, D_{1}}\right)=\frac{4}{3}, \quad \mathbb{E} L\left(J^{0, D_{1}}\right)=2
$$

and

$$
\begin{aligned}
\mathbb{V} L\left(I^{0, D_{1}}\right) & =+\infty \\
\mathbb{V} L\left(K^{0, D_{1}}\right) & =\frac{16}{3}\left(\ln 2-\frac{1}{3}\right), \\
\mathbb{V} L\left(J^{0, D_{1}}\right) & =4 .
\end{aligned}
$$

This are the same values as in the rectangular case.

For the mean values and variances in direction $D_{2}$ we get

$$
\mathbb{E} L\left(I^{0, D_{2}}\right)=6, \quad \mathbb{E} L\left(K^{0, D_{2}}\right)=2, \quad \mathbb{E} L\left(J^{0, D_{2}}\right)=3
$$

and

$$
\begin{aligned}
\mathbb{V} L\left(I^{0, D_{2}}\right) & =+\infty \\
\mathbb{V} L\left(K^{0, D_{2}}\right) & =4(6 \ln 2-1) \\
\mathbb{V} L\left(J^{0, D_{2}}\right) & =9 .
\end{aligned}
$$

For the mean values and variances in direction $D_{3}$ we obtain

$$
\mathbb{E} L\left(I^{0, D_{3}}\right)=12, \quad \mathbb{E} L\left(K^{0, D_{3}}\right)=4, \quad \mathbb{E} L\left(J^{0, D_{3}}\right)=6
$$

and

$$
\begin{aligned}
\mathbb{V} L\left(I^{0, D_{3}}\right) & =+\infty, \\
\mathbb{V} L\left(K^{0, D_{3}}\right) & =16(6 \ln 2-1), \\
\mathbb{V} L\left(J^{0, D_{3}}\right) & =36 .
\end{aligned}
$$

Here we see that the mean values in direction $D_{2}$ are half of the mean values in direction $D_{3}$. This is due to the fact that direction $D_{2}$ is twice more likely to occur for an edge than direction $D_{3}$. Note that there is a similar relation between the mean values in the other directions.

For the mean length of the typical $I-, K$ - and $J$ segment $I^{0}, K^{0}$ and $J^{0}$ we obtain now

$$
\begin{aligned}
\mathbb{E} L\left(I^{0}\right) & =\frac{1}{2} \mathbb{E} L\left(I^{0, D_{1}}\right)+\frac{1}{3} \mathbb{E} L\left(I^{0, D_{2}}\right)+\frac{1}{6} \mathbb{E} L\left(I^{0, D_{3}}\right) \\
& =6 \\
\mathbb{E} L\left(K^{0}\right) & =\frac{1}{2} \mathbb{E} L\left(K^{0, D_{1}}\right)+\frac{1}{3} \mathbb{E} L\left(K^{0, D_{2}}\right)+\frac{1}{6} \mathbb{E} L\left(K^{0, D_{3}}\right) \\
& =2 \\
\mathbb{E} L\left(J^{0}\right) & =\frac{1}{2} \mathbb{E} L\left(J^{0, D_{1}}\right)+\frac{1}{3} \mathbb{E} L\left(J^{0, D_{2}}\right)+\frac{1}{6} \mathbb{E} L\left(J^{0, D_{3}}\right) \\
& =3
\end{aligned}
$$

which confirms the relation in Eq. 8.

\section{ACKNOWLEDGMENTS}

The author would like to thank Joachim Ohser (Darmstadt) for providing the beautiful pictures of the STIT tessellations, Werner Nagel (Jena) for his helpful comments and interesting discussions and the referees for improving considerably the presentation of the manuscript.

This work was supported by the Schweizerischer Nationalfonds grant SNF PP002-114715/1. 


\section{REFERENCES}

Mackisack M, Miles R (1996). Homogeneous rectangular tessellations. Adv Appl Prob 28:993-1013.

Mecke J (2008). Joint distribution of direction and length of the typical $I$-segment in a homogeneous random planar tessellation stable under iteration. Preprint.

Mecke J, Nagel W, Weiss V (2007). Length distributions of edges in planar stationary and isotropic STIT tessellations. Izvestija Akademii Nauk Armenii, Matematika 42:39-60.

Mecke J, Nagel W, Weiss V (2008a). A global construction of homogeneous random planar tessellations that are stable under iteration. Stochastics 80:51-67.

Mecke J, Nagel W, Weiss V (2008b). The iteration of random tessellations and a construction of a homogeneous process of cell divisions. Adv Appl Prob 40:49-59.
Nagel W, Weiss V (2003). Limits of sequences of stationary planar tessellations. Adv Appl Prob 35:123-38.

Nagel W, Weiss V (2005). Crack STIT tessellationscharacterization of stationary random tessellations stable with respect to iteration. Adv Appl Prob 37:85983.

Nagel W, Weiss V (2006). STIT tessellations in the plane. Rendiconti del circulo matematico di Palermo, Serie II, Suppl. 77:441-58.

Stoyan D, Kendall WS, Mecke J (1995). Stochastic Geometry and its Applications. 2nd Ed. Chichester: Wiley.

Thäle C (2008). $K$ - and $J$-segments in homogeneous planar STIT tessellations. Preprint.

Thäle C (2009). On the length of certain segments in higher dimensional STIT tessellations. Preprint. 